\title{
A silent trio: giant descending aortic aneurysm combined with coarctation and persistent left brachiocephalic truncus
}

\author{
Maksim Basho ${ }^{1}$, Deniona Nunci ${ }^{1}$, Gentian Vyshka ${ }^{2}$, and Edvin Prifti ${ }^{1}$ \\ ${ }^{1}$ University Hospital Center 'Mother Teresa', Tirana \\ ${ }^{2}$ Faculty of Medicine, University of Medicine
}

January 5, 2021

\begin{abstract}
We report the case of an undiagnosed descending aortic aneurysm, combined with coarctation and persistent left brachiocephalic truncus in a 59-years old male. This case underscores the increased necessity for aortic imaging while facing a poorly controlled hypertension, erroneously considered as of essential origin.
\end{abstract}

\section{A silent trio: giant descending aortic aneurysm combined with coarctation and persistent left brachiocephalic truncus}

Keywords: giant aneurysm; descending aorta; left brachiocephalic truncus; coarctation.

\section{Key clinical message:}

We report a case of an undiagnosed descending aortic aneurysm, combined with coarctation and persistent left brachiocephalic truncus in a 59-years old male. It highlights the necessity for aortic imaging, when facing a poorly controlled hypertension.

\section{CLINICAL IMAGES}

A 59-year-old Albanian man presented at for radiological evaluation following a two -week period of effort dyspnea. He had a poorly controlled hypertension and was a lifetime heavy smoker (more than twenty cigarettes daily). During a cardiological consultancy, his blood pressure was $170 / 120 \mathrm{mmHg}$, with a heart rate of 110 beats per minute. A diastolic murmur was the only finding in the auscultation, and electrocardiogram was considered within normality. A CT angiogram of the thorax was performed the same day, with impressive images of a giant descending aortic aneurysm that reached a maximum of 9,8 centimeters in transverse diameter (Figure 1a and 1b).

$<$ Figure 1a-b here $>$

A dissecting flap of more than four centimeters was as well visualized. Proximally to the dilated portion, the aortic arch was severely coarcted, with a narrowed opening of about 6,8 millimeters in the transverse diameter. Hypertrophic intercostal arteries were present at the sagittal reconstructed CT images. The patient was never diagnosed previously for coarctation; furthermore, the axial CT images showed the presence of left brachiocephalic artery (Figure $2 \mathrm{a}$ and $2 \mathrm{~b}$ ).

$<$ Figure 2a-b here $>$

Giant asymptomatic aortic aneurysms are rarely reported, but still present and therefore, a cause of major concern in emergency medicine $[1,2]$.

\section{REFERENCES}


Shah P, Gupta N, Goldfarb I, Shamoon F. Giant dissecting aortic aneurysm in an asymptomatic young male. Case Rep Vasc Med. 2015;2015:958464.

Okura T, Kitami Y, Takata Y, Fukuoka T, Arimitsu J, Hiwada K. Giant unruptured aneurysm of the thoracic aorta-a case report. Angiology. 1999 Oct;50(10):865-9.

\section{FIGURE LEGENDS}

Figure 1a: Descending aorta presented extremely dilated, with a transverse diameter of 9,78 cm (markers). 1b: Angio CT reconstruction images showing the giant aneurysm.

Figure 2a: Sagittal thorax reconstructed CT images showing the presence of hypertrophic intercostal arteries (arrow) as well as the giant aneurysm with a dissected flap (asterisk).2b: Presence of right and left brachiocephalic trunci, with respective transverse diameters in centimeters.

\section{ACKNOWLEDGMENTS}

Published with written consent of the patient.

\section{CONFLICT OF INTEREST}

None declared.

\section{AUTHOR CONTRIBUTIONS}

$\mathrm{MB}, \mathrm{DN}, \mathrm{GV}$ and EP: involved in manuscript writing, data collection, and literature reviewing.

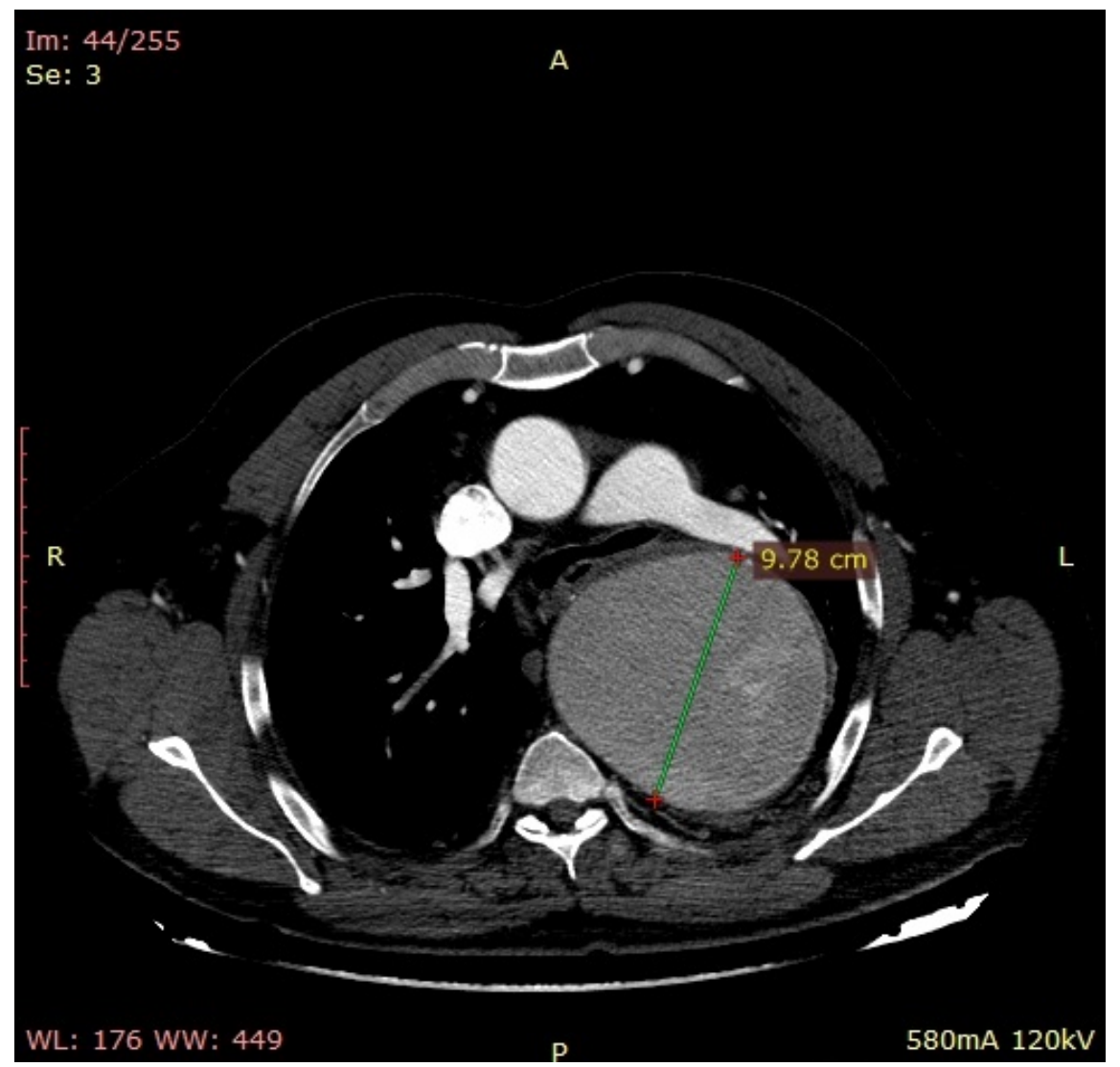




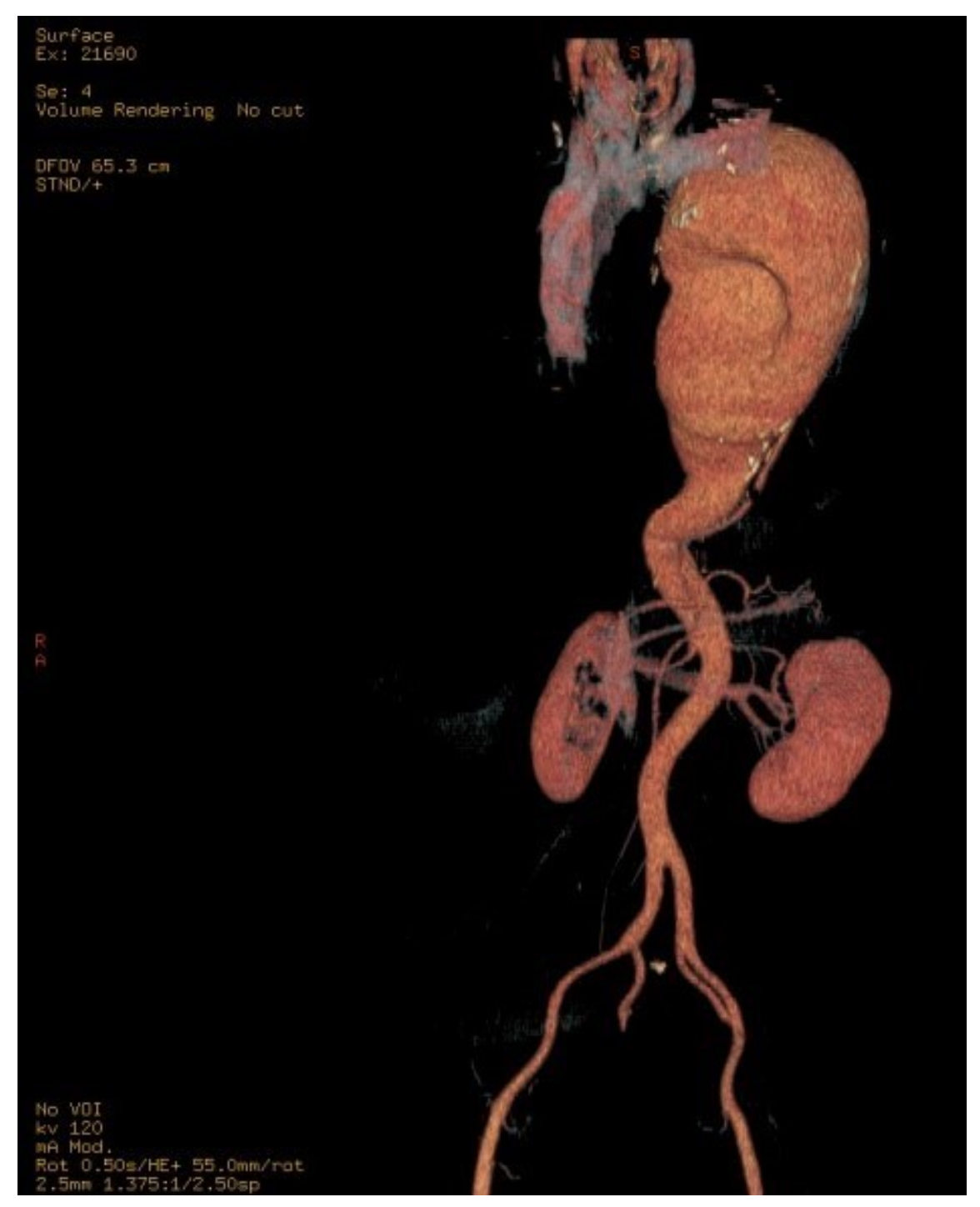




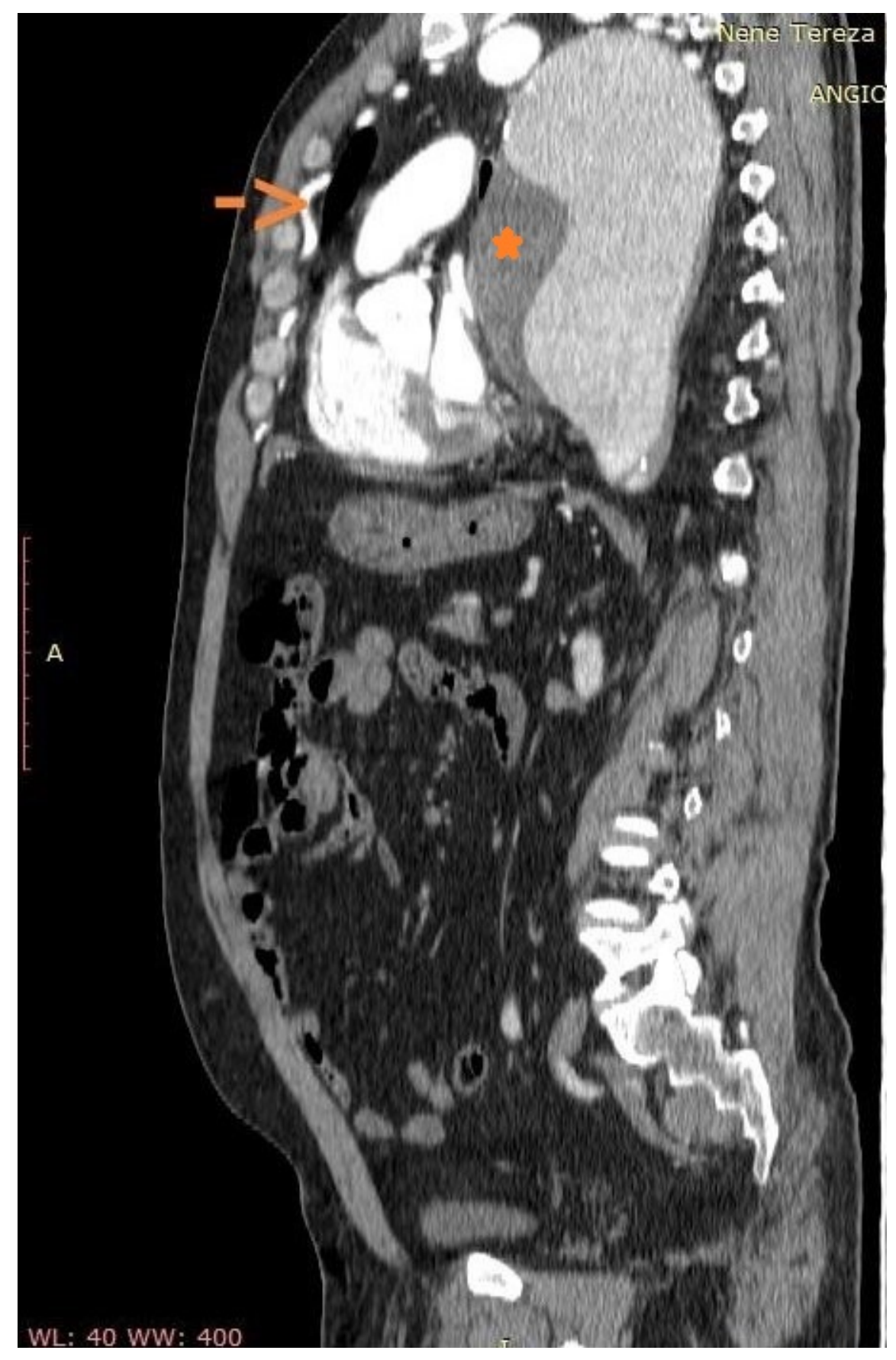




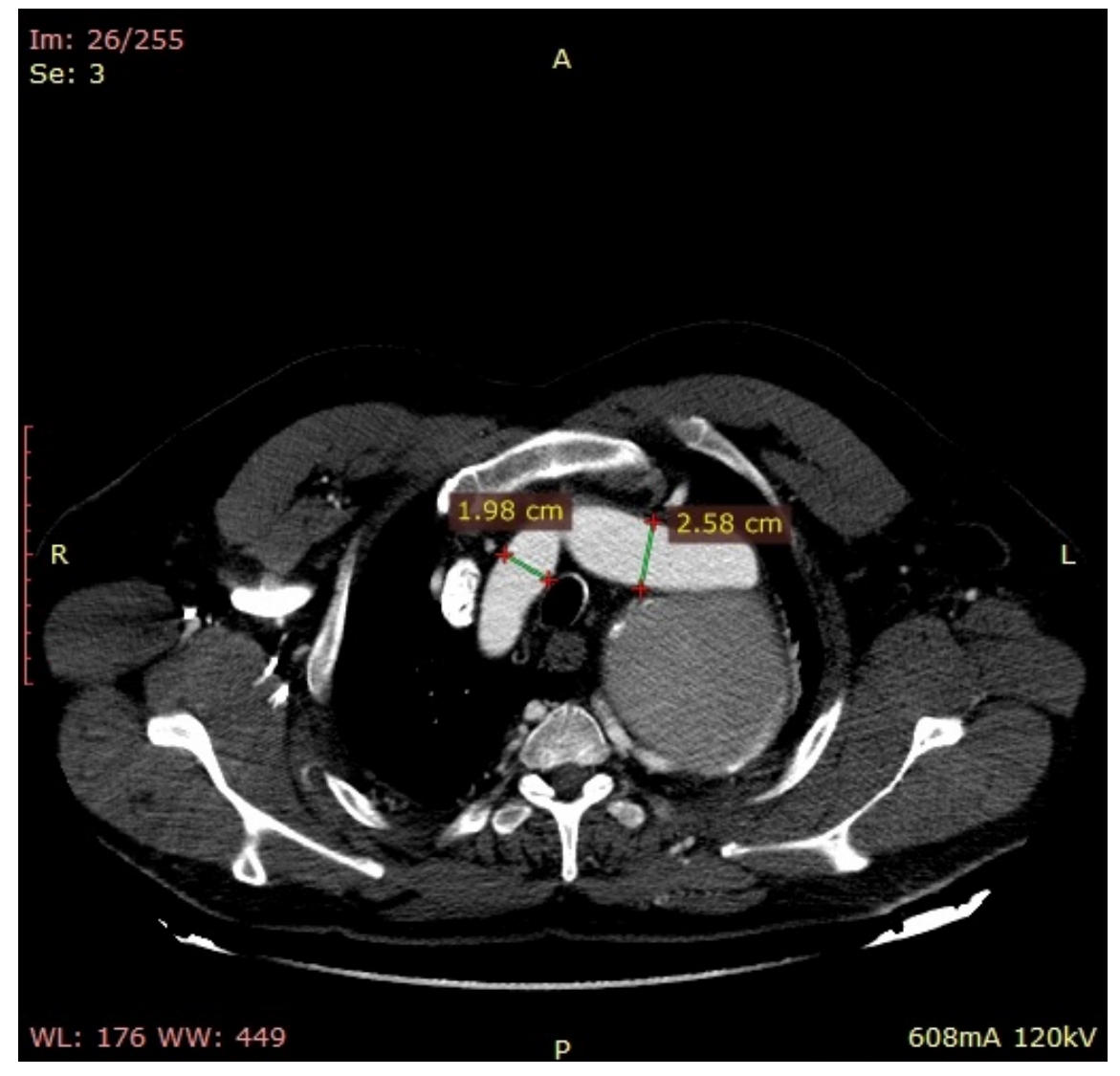

